\title{
Identifying packing features of atoms with distinct dynamic behaviors in metallic glass by machine- learning method
}

\author{
Yicheng $\mathrm{Wu}^{1,2,3}$, Wei-Hua Wang ${ }^{1,3,5}$, Pengfei Guan ${ }^{2^{*}}$ and Haiyang Bai ${ }^{1,3,4^{*}}$
}

In crystalline solids, plastic deformation is determined by the motion of structural defects, particularly dislocations. As for glassy liquids and disordered solids, it has long been assumed that localized structural rearrangements induced by mechanical load or thermal stimulus also occur at localized defects [1-3]. Metallic glasses have many promising applications as structural materials due to the unique long-range disordered atomic structure [4]. However, such structural disorder makes it overwhelmingly difficult to build a causal link between structure and plastic rearrangements [5-7]. The most promising structural indicators acting as structural defects are soft spots based on the soft vibrational modes $[8,9]$, and volume flexibility [10]. Nevertheless, these indicators are based on the measurement of specific physical quantities and require detailed knowledge of interparticle interactions. Geometrically unfavored motifs (GUMs) and local fivefold symmetry (LFFS) $[6,7,11,12]$, as purely structural indicators, can partially predict the nature of localized atomic rearrangements. However, metallic liquids or glasses have diverse atomic clusters, and thus it is not easy to distinguish which GUMs would be the ones that actually facilitate relaxation events. Recent work showed that such simple short-range order parameter as LFFS is not a sound structural fingerprint of dynamics of glasses because the spatial autocorrelation length of LFFS is only $1.2 \AA$, much shorter than the common spatial correlation range of approximately $2.2 \AA$ [13]. Very recently, Cubuk et al. [14-20] successfully proposed a purely structural parameter, softness, by combining symmetry functions as structural representa- tions with machine learning (ML) to capture the plasticity and dynamics in simple disorder systems with LennardJones interaction. However, in metallic glassy systems with many-body interaction and composition-sensitive properties, the purely structural descriptor of dynamics still remains a mystery. Thus, it is of particular interest to investigate the structure-dynamics correlation in metallic glasses by ML methods.

In this work, we employ the ML method to build a predictive model in a simulated $\mathrm{Cu}_{50} \mathrm{Zr}_{50}$ metallic glass. The atomic softness defined by this predictive model is found to be strongly correlated with the propensity for structural rearrangements. The atoms predicted with positive or negative softness can be classified as "liquidlike" or "solid-like", respectively. By systematically analyzing the structure of atoms with distinct softness, we observe that the structural difference between these two classes of atoms is dominated by the short-range structure within the first-neighbor shell, associated with non-negligible structure in the medium-range. Moreover, a characteristic distance can be extracted to distinguish the short-range packing features of the two kinds of atoms. Compared with the "solid-like" atoms, the "liquid-like" ones present more dense packing inside the characteristic distance but more loose packing beyond the characteristic distance. It indicates more anisotropic local geometrical packing feature of the "liquid-like" atoms, and provides useful hints about the structure-dynamics correlation in metallic glasses.

A prototypical binary system $\mathrm{Cu}_{50} \mathrm{Zr}_{50}$ with the embedded atom method (EAM) potential is employed in this

\footnotetext{
${ }^{1}$ Institutes of Physics, Chinese Academy of Sciences, Beijing 100190, China

${ }^{2}$ Beijing Computational Science Research Center, Beijing 100193, China

${ }^{3}$ School of Physical Sciences, University of Chinese Academy of Sciences, Beijing 100049, China

${ }^{4}$ Center of Materials Science and Optoelectronics Engineering, University of Chinese Academy of Sciences, Beijing 100049, China

${ }^{5}$ Songshan Lake Materials Laboratory, Dongguan 523808, China

*Corresponding authors (emails: pguan@csrc.ac.cn (Guan P); hybai@iphy.ac.cn (Bai H))
} 
work [21], which is selected for its superior glass-forming ability and simple constituents. The system consists of $N$ $=4000$ atoms. The sample is first equilibrated at $2000 \mathrm{~K}$ for $2 \mathrm{~ns}$, and then quenched with a cooling rate of $10^{10} \mathrm{~K} \mathrm{~s}^{-1}$, during which the external pressure is adjusted to zero within the constant number, pressure and temperature (NPT) ensemble. The glass transition temperature $T_{\mathrm{g}}$ is around $750 \mathrm{~K}$. Simulations are done using LAMMPS [22] with a Nose-Hoove thermostat [23] and a time step of $\delta t=0.002 \mathrm{ps}$. The glassy liquid at $800 \mathrm{~K}$ is first equilibrated in the NPT ensemble for $1 \mathrm{~ns}$, and the configurations are then collected with a time interval $\Delta t=$ $1 \mathrm{ps}$ in the constant number, volume and temperature (NVT) ensemble for structure and dynamics analyses.

To explore the correlation between local structure and atomic rearrangement by ML methods, we first need to define the structural rearrangement, thus identifying the rearranging and non-rearranging atoms. In the supercooled regime, the atom constantly vibrates locally in the cage formed by its neighbors and rarely escapes from the cage with a large displacement. To identify a rearrangement event in the three-dimensional quiescent system, we use a "hop" indicator function, $p_{\text {hop }}$, which was introduced by Candelier et al. [24,25]. To define $p_{\text {hop }}(i ; t)$ for atom $i$ at time $t$, we first specify two time intervals $A=$ $\left[t-t_{\mathrm{R}} / 2, t\right]$ and $B=\left[t, t+t_{\mathrm{R}} / 2\right]$. The "hop" indicator function then can be written as

$p_{\text {hop }}(i ; t)=\sqrt{\left\langle\left(\boldsymbol{r}_{i}-\left\langle\boldsymbol{r}_{i}\right\rangle_{B}\right)^{2}\right\rangle_{A}\left\langle\left(\boldsymbol{r}_{i}-\left\langle\boldsymbol{r}_{i}\right\rangle_{A}\right)^{2}\right\rangle_{B}}$,

where \langle\rangle$_{A}$ and \langle\rangle$_{B}$ are averages over the intervals $A$ and $B$, respectively. If no rearrangement takes place between intervals $A$ and $B$, then $\left\langle\boldsymbol{r}_{i}\right\rangle_{A}$ and $\left\langle\boldsymbol{r}_{i}\right\rangle_{B}$ are nearly equal and $p_{\text {hop }}$ will be a small value. If a rearrangement does occur, the mean positions will change appreciably and $p_{\text {hop }}$ will attain a significantly large value. The hop time parameter, $t_{\mathrm{R}}$, used in the "hop" indicator function should be approximately the time it takes for the system to complete a rearrangement $[15,20,25]$. Here we take $t_{R}=$ $10 \mathrm{ps}$ to be at the end of the plateau-like region of the mean square displacement (MSD) data shown in the Supplementary information (Fig. S1). The distance and $p_{\text {hop }}$ of an atom as a function of time can be seen in Fig. 1a and $b$, respectively. In the beginning, the atom locally vibrates within a cage, and the corresponding value of $p_{\text {hop }}$ is small. When the atom hops to a new cage with a large displacement, the value of $p_{\text {hop }}$ increases accordingly. Therefore, the "hop" indicator function can well describe the atomic rearrangement. We define all rearrangements to be those events with $p_{\text {hop }}>p_{c}$. The $p_{c}$ is chosen such that $1 \%$ of the atoms from each species have
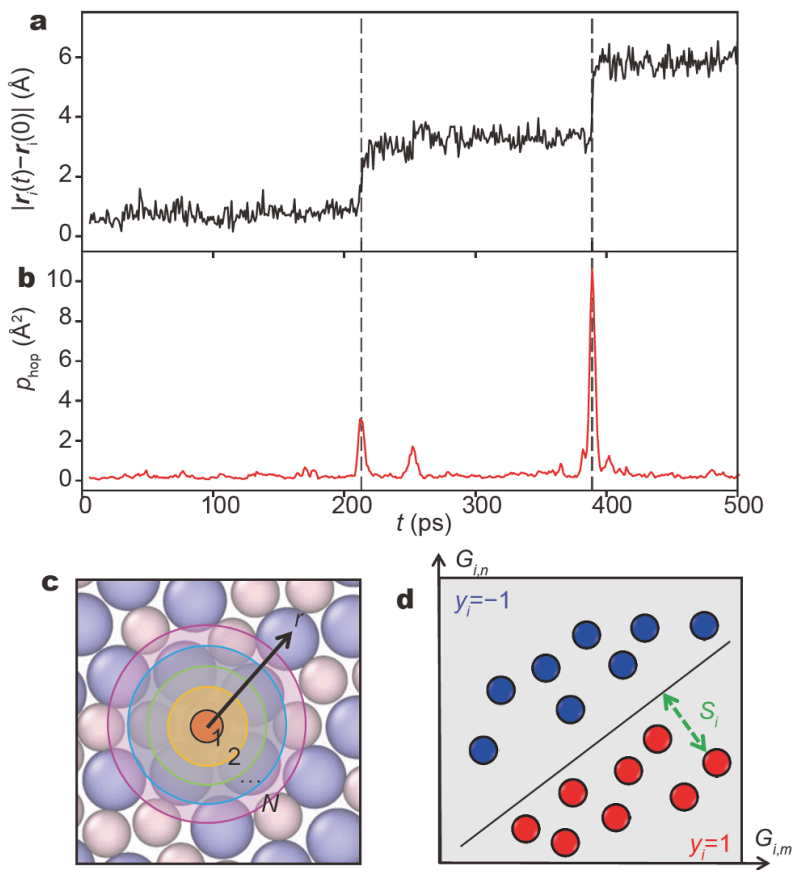

Figure 1 (a) The distance that an atom has moved from its initial position and (b) the corresponding "hop" indicator $p_{\text {hop }}$ over time. Successive rearrangement events can be seen. (c) A schematic of the function $G_{i}(r)$ for several different values of $r$. (d) A schematic showing how the local structure of an atom is represented by $\boldsymbol{G}_{i}$. We can find a hyperplane (black line) separating rearranging atoms $\left(y_{i}=1\right)$ from stable ones $\left(y_{i}=-1\right)$ by SVM. Once the hyperplane is established, the softness $S_{i}$ can be defined as the distance of atom $i$ embedded into the highdimensional space to the hyperplane.

gone through a rearrangement, although the specific choice of $p_{c}$ does not affect our results qualitatively. The $p_{\text {hop }}$ distributions of species $\mathrm{Cu}$ and $\mathrm{Zr}$ atoms differ significantly (see Fig. S2), and thus these two species are treated separately throughout the analysis. In this work, all results here are for the central atoms of species $\mathrm{Cu}$ only, but the results also hold for species $\mathrm{Zr}$.

To employ ML methods, characterization of the local structure of each atom is needed. There are a wide variety of methods that can be used $[15,26,27]$. In order to more easily interpret the ML models and extract physical insights from them, we describe the local structure by the radial symmetry function $[16,17]$

$G_{i}^{X}(r)=\sum_{j \in X} e^{-\frac{1}{2 \delta^{2}}\left(r-R_{i j}\right)^{2}}$,

where $R_{i j}$ is the distance between atoms $i$ and $j$, and $X \in\{\mathrm{Cu}, \mathrm{Zr}\}$ refers to the species of atoms near atom $i$ within $R_{i j}<R_{\mathrm{c}}=10 \AA$. As shown in Fig. $1 \mathrm{c}, G_{i}{ }^{X}(r)$ essentially reveals the Gaussian-smeared radial density of 
species $X$ at each $r$. In practice, we discretize the set of radii into $\boldsymbol{r}_{n}=r_{0}+n \delta$ and consider the vector

$$
\boldsymbol{G}_{i}=\left(G_{i}^{\mathrm{Cu}}\left(r_{1}\right), \ldots, G_{i}^{\mathrm{Cu}}\left(r_{N}\right), G_{i}^{\mathrm{Zr}}\left(r_{1}\right), \ldots, G_{i}^{\mathrm{Zr}}\left(r_{N}\right)\right) \text {. }
$$

In this work, we keep $\delta=0.1 \AA, r_{0}=2 \AA$, and $N=80$ fixed. Therefore, we characterize the local structure around the target atom $i$ by 160 structural descriptors $G_{i, \alpha}$ (where $\alpha$ indexes $1,2, \ldots, 160$ ). We note that the different choices of $\delta$ and $N$ only bring trifling impact on the results so long as $r_{n}$ is large enough to include several neighbor shells. $\boldsymbol{G}_{i}$ of an atom contains details of local geometrical structure across several shells, as well as chemical features. Thus, we expect that $\boldsymbol{G}_{i}$ can sufficiently capture the local structure of individual atom.

Having defined rearranging and non-rearranging atoms through $p_{\text {hop }}$ and characterized local structure of each atom through $\boldsymbol{G}_{i}$, we now use an ML method called support vector machine (SVM) [28] to find function of features (in this case about $\boldsymbol{G}_{i}$ ) that optimally separates points in different classes (in this case about rearranging and non-rearranging atoms). The SVM algorithm is implemented using the LIBSVM package [29]. Firstly, we select a training set with $N$ atoms that are about to rearrange in the next $1 \mathrm{ps}$, each labeled as $y_{i}=1$, and equal atoms that will not rearrange, each labeled as $y_{i}=-1$. Therefore, a training set of $2 \mathrm{~N}$ atoms can be written as $\left\{\left(\boldsymbol{G}_{1}, y_{1}\right), \ldots,\left(\boldsymbol{G}_{2 N}, y_{2 N}\right)\right\}$. We next use SVM with a linear kernel to construct a hyperplane defined by the hyperplane's normal $\boldsymbol{w}$ and the bias $b$, that best divides atoms with $y_{i}=1$ from those with $y_{i}=-1$ in the training set. Once the hyperplane has been constructed, we then employ this hyperplane on the test set. For atom $i$ in the test set, its local structure can be described by $\boldsymbol{G}_{i}$, the softness $S_{i}$ is then defined as $S_{i}=\boldsymbol{w} \cdot \boldsymbol{G}_{i}+b$ [15], which is shown schematically in Fig. 1d. The atom will be classified as "liquid-like" (prone to rearrangement) if $S_{i}>0$, and as "solid-like" (resistant to rearrangement) if $S_{i}<0$.

We choose the training set with 10,000 rearranging atoms, each labeled as $y_{i}=1$, and equal non-rearranging atoms, each labeled as $y_{i}=-1$. After the ML training, the hyperplane, acting as the ML model, is constructed. The hyperplane normal $\omega(r)$ for $\mathrm{Cu}-\mathrm{Cu}$ and $\mathrm{Cu}-\mathrm{Zr}$ pairs obtained from the SVM is shown in Fig. 2a and b, respectively. Since the softness is defined as integrating the local structure of $G_{i}(r)$ weighted by $\omega(r)$, a large absolute value of $\omega(r)$ within short-range indicates the short-range order will impose a great effect on softness. Moreover, $\omega(r)$ beyond short-range distance is nontrivial, especially for $\mathrm{Cu}-\mathrm{Cu}$ pairs, which implies that the medium-range structures play a nonnegligible role in determining soft- ness. We then construct a test set of $2 \times 10^{6}$ atoms. These atoms are aggregated over every snapshot of the simulation, and the softness of all atoms in the test set is calculated using the machine-learned hyperplane.

To investigate the effectiveness of softness at predicting rearrangements, we first check the relation between softness and $p_{\text {hop. }}$ In Fig. $2 c$, we plot the average softness with $p_{\text {hop }}$. There is a clear trend that the higher the softness, the lager the $p_{\text {hop. }}$. Furthermore, we investigate the probability, $P_{\mathrm{R}}$, that atoms will rearrange as a function of softness. $P_{\mathrm{R}}$ is calculated as the fraction of atoms with a softness that will undergo rearrangements. As shown in Fig. 2d, the probability of rearrangement of high-softness atoms is nearly two orders of magnitude larger than their low-softness counterparts, indicating the robustness of the softness in predicting rearrangements. In Fig. 2e, the contoured map illustrates the spatial distribution of the softness on a slice with a thickness of $10 \AA$. The locations of the white spots superimposed on the contour denote the spatial information of rearranging atoms. Obviously, the rearrangements have a high propensity to originate from those "liquid-like" regions with $S>0$. Since atoms with higher softness are more prone to rearrangement, it is necessary to check whether the size of rearrangements is limited by the spatial extent of high-softness regions. Therefore, we first investigate the spatial correlation function of the softness field [19]

$$
C(r)=\langle\delta S(0) \delta S(r)\rangle=\frac{\langle S(0) S(r)\rangle-\langle S(0)\rangle^{2}}{\left\langle S^{2}(0)\right\rangle-\langle S(0)\rangle^{2}}
$$

As shown in Fig. 2f, we find that $\langle\delta S(0) \delta S(r)\rangle$ exhibits approximately exponential decay, and the red dashed line is fitted by $\langle\delta S(0) \delta S(r)\rangle=A e^{-r / \xi_{S}}$ with $\xi_{S} \approx 2.52 \AA$. This length scale is commensurate with the spatial correlation length previously identified in metallic glassy systems [13], implying that softness is also a good structural descriptor to predict the dynamic feature of metallic glasses. To measure the spatial extent of rearrangements, we then consider the spatial correlation of $p_{\text {hop }}$. As with softness, we find that the correlation $\left\langle\delta p_{\text {hop }}(0) \delta p_{\text {hop }}(r)\right\rangle$ decays exponentially with $\xi_{R} \approx 2.55 \AA$ A. Thus, we can conclude that the characteristic size of localized rearrangements is encoded in the size of spatial correlation of softness in metallic glassy system. This observation is in excellent agreement with the earlier results in Kob-Andersen model systems [16].

The above results demonstrate that the softness is effective in predicting the rearrangement, indicating that there is a strong structural component to rearrangement. 

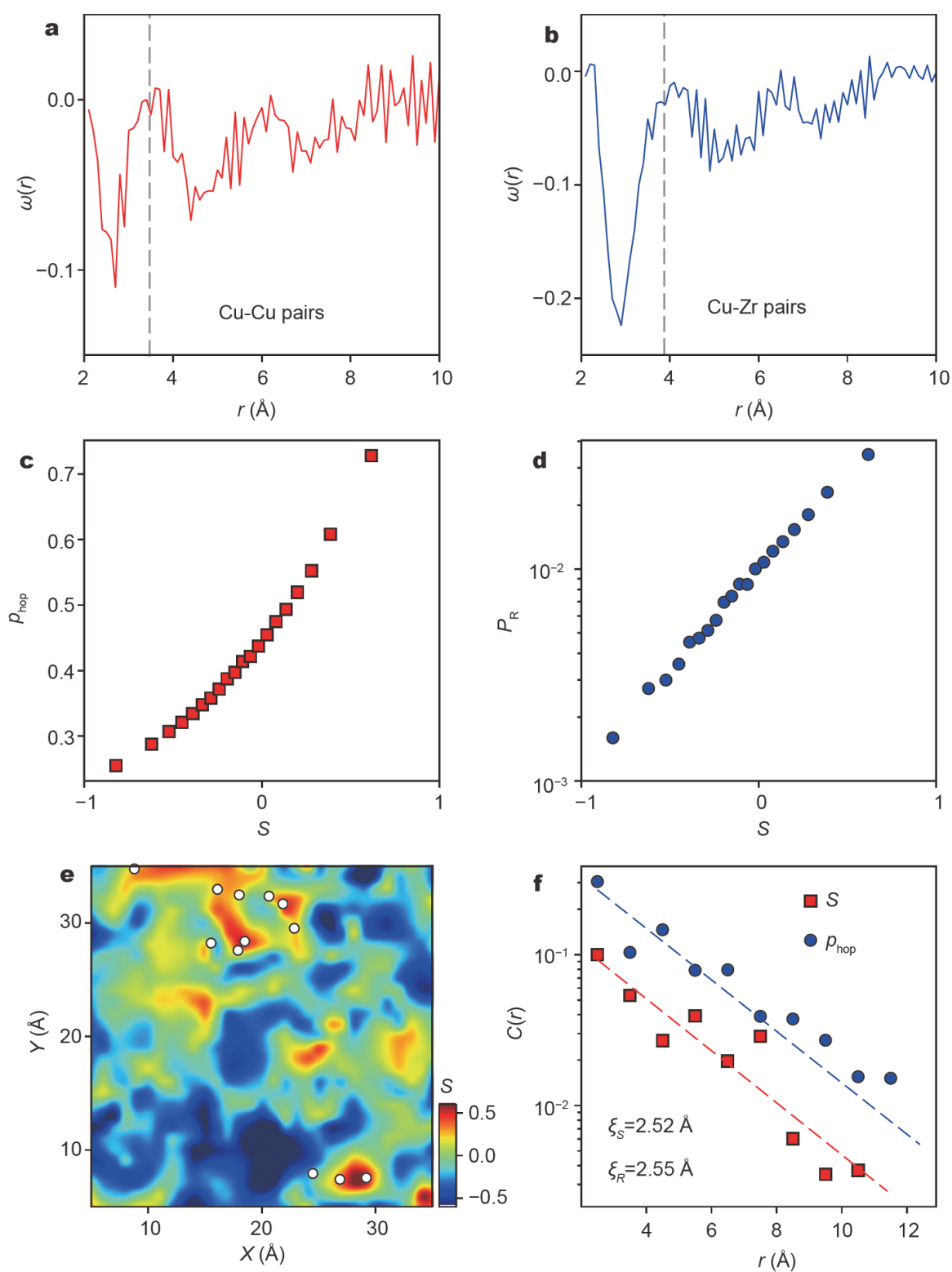

Figure $2(\mathrm{a}, \mathrm{b})$ The hyperplane normal $\omega(r)$ for $\mathrm{Cu}-\mathrm{Cu}$ and $\mathrm{Cu}-\mathrm{Zr}$ pairs, respectively. The black dashed lines denote the first trough position of the $\mathrm{RDF}$ of the system for $\mathrm{Cu}-\mathrm{Cu}$ and $\mathrm{Cu}-\mathrm{Zr}$ pairs, respectively. (c) Correlation between the average $p_{\text {hop }}$ with softness. Each data point is the average for $5 \%$ of all of the atoms, sorted in the order of increasing softness. (d) The probability that atoms will rearrange as a function of their softness. Each data point is the average for $5 \%$ of all of the atoms, sorted in the order of increasing softness. (e) The contour map denoting the softness field in the slice of $10 \AA$ thick. The locations of the rearrangements are indicated by the white spots. (f) The spatial correlation functions for softness (red) and rearrangement (blue). The dashed lines are fits to $A e^{-r / \xi_{S}}$ with $\xi_{S} \approx 2.52 \AA$ for softness and $A e^{-r / \xi_{R}}$ with $\xi_{R} \approx 2.55 \AA$ for rearrangement.

To illustrate this, we refer to the radial distribution function $(\mathrm{RDF})$ of the "liquid-like" $(S>0)$ and the "solidlike" $(S<0)$ atoms. When the system is isotropic, the structure function $G_{i}{ }^{X}(r)$ is related to the RDF [14]

$g_{A-X}(r)=\lim _{\delta \rightarrow 0}\left\langle G_{i}^{X}(r)\right\rangle /\left(4 \pi r^{2}\right)$,

where $A$ and $X$ are species of the central atom and neighboring atom, respectively. As shown in Fig. 3a and $\mathrm{b}$, we notice that the "liquid-like" (or "solid-like") atoms classified by the hyperplane and the rearranging (or nonrearranging) ones have similar RDFs, implying that the hyperplane learned from the SVM can reliably distinguish rearranging atoms from stable ones. Comparing $g(r)$ of the "liquid-like" with the "solid-like" atoms for $\mathrm{Cu}-\mathrm{Cu}$ 

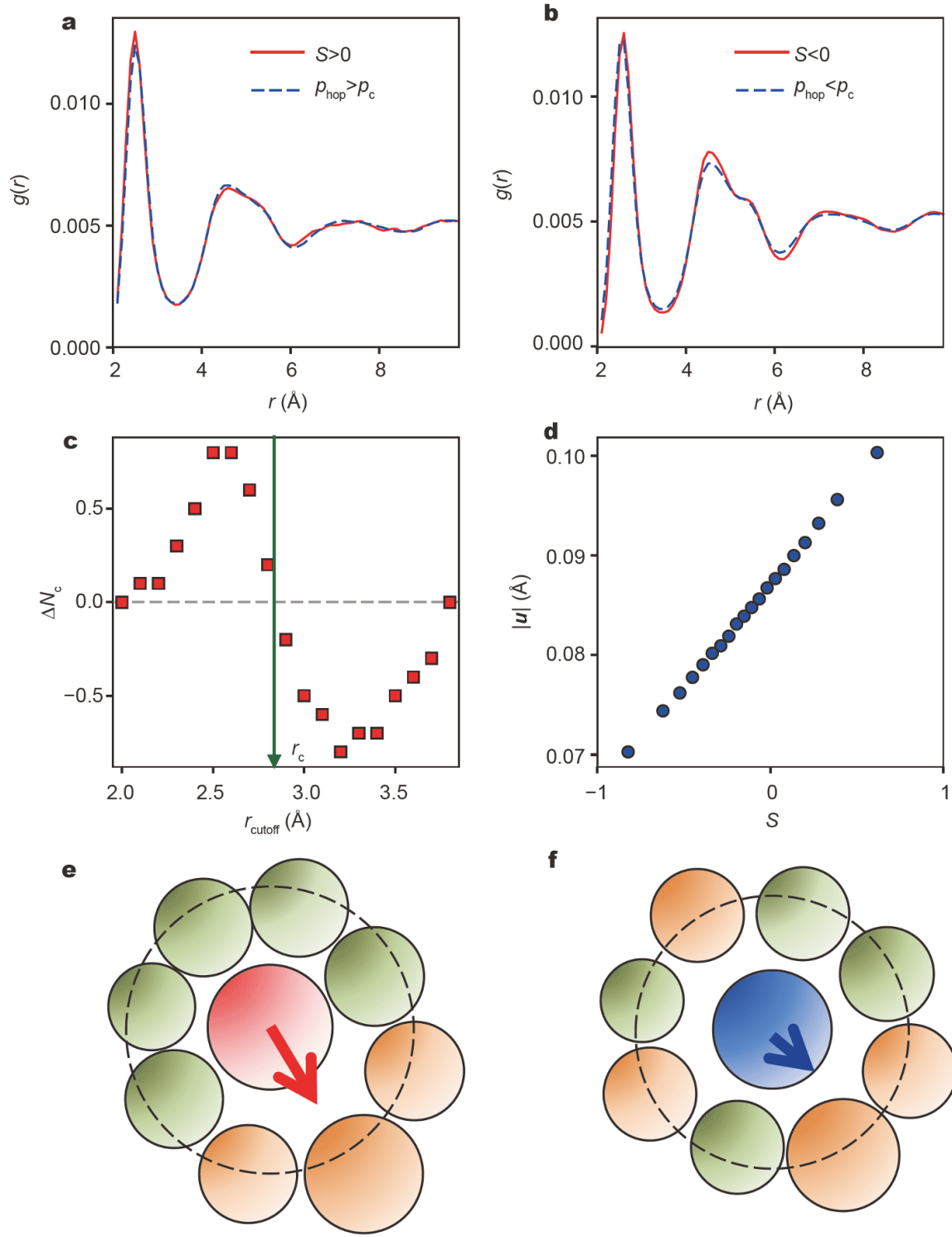

$\mathbf{f}$

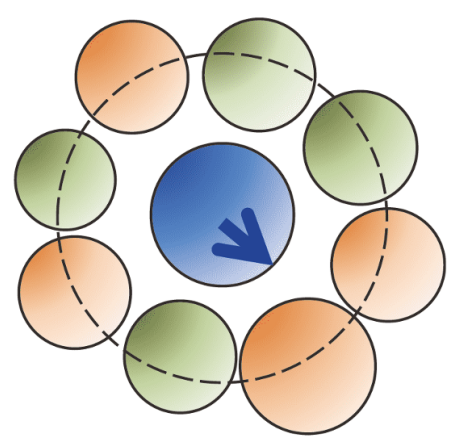

Figure 3 (a, b) The RDFs of Cu-Cu pairs averaged over "liquid-like" atoms with $S>0$ (red line) versus rearranging atoms with $p_{\text {hop }}>p_{c}$ (blue dashed line) and "solid-like" atoms with $S<0$ (red line) versus non-rearranging atoms with $p_{\text {hop }}<p_{\mathrm{c}}$ (blue dashed line), respectively. (c) The difference of coordination number $\Delta N_{\mathrm{c}}$ as a function of $r_{\text {cutoff }}$ between "liquid-like" and "solid-like" atoms. The green arrow denotes the characteristic length of $r_{\mathrm{c}}=$ $2.85 \AA$. (d) Correlation between the average local configurational anisotropy $|\boldsymbol{u}|$ with softness. Each data point is the average for $5 \%$ of all of the atoms, sorted in the order of increasing softness. (e, f) The schematic of local geometry for "liquid-like" (red) and "solid-like" (blue) atoms, respectively. The black dashed circles denote the range of characteristic length $r_{\mathrm{c}}$. The length of arrows denotes the magnitude of the local configurational anisotropy $|\boldsymbol{u}|$. Atoms inside the circular region of the radius $r<r_{\mathrm{c}}$ are colored as green, while atoms outside the circular region are colored as orange.

pairs, we find that the structural differences between them are beyond the first shell. The obvious splitting of the second peak suggests the enhanced medium-range order for the "solid-like" atoms. The similar results can also be found for $g(r)$ of $\mathrm{Cu}-\mathrm{Zr}$ pairs shown in Fig. S3.

To fully understand the local environment of the "liquid-like" and the "solid-like" atoms, we next calculate the coordination number, $N_{c}$, of these two groups of atoms. The difference of $N_{c}$ between them, $\Delta N_{c}=N_{c}(S>$ $\left.0, r_{\text {cutoff }}\right)-N_{c}\left(S<0, r_{\text {cutoff }}\right)$, as a function of $r_{\text {cutoff }}$ is shown in Fig. 3c. It shows that there exists a clear characteristic length $r_{\mathrm{c}}=2.85 \AA$ that is approximately equal to the first peak position of the RDF of the system, within the first neighbor shell. The "liquid-like" atoms have more 
neighbors when $r_{\text {cutoff }}$ is below $r_{c}$, while the result is opposite when $r_{\text {cutoff }}$ is above $r_{c}$, suggesting that the "liquidlike" atoms are tightly packed within $r_{\mathrm{c}}$ but loosely packed beyond $r_{\mathrm{c}}$. This observation is further confirmed in another metal-metal glass $\mathrm{Ni}_{50} \mathrm{Nb}_{50}$ [30] and a metal-metalloid glass $\mathrm{Ni}_{80} \mathrm{P}_{20}$ [31], indicating the generality of this finding among metallic glasses (see Fig. S4). Furthermore, we analyze the local configurational anisotropy, $\left|\boldsymbol{u}_{i}\right|$, defining the deviation from the center of atom $i$ to the centroid of its corresponding Voronoi cell [32-34]. For atoms in full icosahedra, the center of the atom and the corresponding Voronoi cell centroid coincide and $\left|\boldsymbol{u}_{i}\right|=0$. However, for atoms in distorted polyhedra, the atom's center deviates from the centroid of corresponding Voronoi polyhedron. Therefore, the local configurational anisotropy signifies the degree of symmetry breaking of atomic environment and thus the propensity for vibrations [32-36]. As shown in Fig. 3d, $|\boldsymbol{u}|$ linearly increases with softness. It implies that the "liquid-like" atoms with larger value of softness have more anisotropic structure and thereby are more likely to rearrange. From the above, we can depict the local geometry of the "liquid-like" and the "solid-like" atoms shown in Fig. 3e and f, respectively. As compared with "solid-like" atoms, "liquid-like" counterparts are closely packed within the characteristic range $r_{\mathrm{c}}$, but have more local void spaces beyond the characteristic range $r_{\mathrm{c}}$. Moreover, "liquid-like" atoms present higher degree of distortion in their atomic environment, indicating a high propensity for rearrangements.

In summary, we have presented a novel ML approach to identify the atoms prone to rearrangements in the $\mathrm{Cu}_{50} \mathrm{Zr}_{50}$ metallic glassy system. We find that the "liquidlike" and the "solid-like" atoms classified by machinelearned hyperplane display distinct dynamic features. Moreover, we observe a characteristic length $r_{\mathrm{c}}$ within the first neighbor shell differentiating the short-range packing features of these two classes of atoms. The "liquidlike" atoms present more tight packing within $r_{c}$, but more loose packing beyond $r_{\mathrm{c}}$. In addition, the "liquidlike" atoms have more anisotropic atomic environment, which promotes rearrangements. Our findings provide comprehensive insights into the structure-dynamics relationship of metallic glassy systems.

\section{Received 20 December 2020; accepted 23 January 2021;} published online 18 March 2021

1 Argon AS. Plastic deformation in metallic glasses. Acta Metall, 1979, 27: $47-58$

2 Falk ML, Langer JS. Dynamics of viscoplastic deformation in amorphous solids. Phys Rev E, 1998, 57: 7192-7205

3 Spaepen F. A microscopic mechanism for steady state inhomogeneous flow in metallic glasses. Acta Metall, 1977, 25: 407415

4 Telford M. The case for bulk metallic glass. Mater Today, 2004, 7: 36-43

5 Schuh CA, Hufnagel TC, Ramamurty U. Mechanical behavior of amorphous alloys. Acta Mater, 2007, 55: 4067-4109

6 Cheng YQ, Ma E. Atomic-level structure and structure-property relationship in metallic glasses. Prog Mater Sci, 2011, 56: 379-473

7 Ma E. Tuning order in disorder. Nat Mater, 2015, 14: 547-552

8 Widmer-Cooper A, Perry H, Harrowell P, et al. Irreversible reorganization in a supercooled liquid originates from localized soft modes. Nat Phys, 2008, 4: 711-715

9 Ding J, Patinet S, Falk ML, et al. Soft spots and their structural signature in a metallic glass. Proc Natl Acad Sci USA, 2014, 111: 14052-14056

10 Ding J, Cheng YQ, Sheng H, et al. Universal structural parameter to quantitatively predict metallic glass properties. Nat Commun, 2016, 7: 13733

11 Peng HL, Li MZ, Wang WH. Structural signature of plastic deformation in metallic glasses. Phys Rev Lett, 2011, 106: 135503

$12 \mathrm{Hu}$ YC, Li FX, Li MZ, et al. Five-fold symmetry as indicator of dynamic arrest in metallic glass-forming liquids. Nat Commun, 2015, 6: 8310

13 Wei D, Yang J, Jiang MQ, et al. Revisiting the structure-property relationship of metallic glasses: Common spatial correlation revealed as a hidden rule. Phys Rev B, 2019, 99: 014115

14 Cubuk ED, Schoenholz SS, Rieser JM, et al. Identifying structural flow defects in disordered solids using machine-learning methods. Phys Rev Lett, 2015, 114: 108001

15 Schoenholz SS, Cubuk ED, Sussman DM, et al. A structural approach to relaxation in glassy liquids. Nat Phys, 2016, 12: 469-471

16 Cubuk ED, Schoenholz SS, Kaxiras E, et al. Structural properties of defects in glassy liquids. J Phys Chem B, 2016, 120: 6139-6146

17 Sussman DM, Schoenholz SS, Cubuk ED, et al. Disconnecting structure and dynamics in glassy thin films. Proc Natl Acad Sci USA, 2017, 114: 10601-10605

18 Schoenholz SS, Cubuk ED, Kaxiras E, et al. Relationship between local structure and relaxation in out-of-equilibrium glassy systems. Proc Natl Acad Sci USA, 2017, 114: 263-267

19 Cubuk ED, Ivancic RJS, Schoenholz SS, et al. Structure-property relationships from universal signatures of plasticity in disordered solids. Science, 2017, 358: 1033-1037

20 Ma X, Davidson ZS, Still T, et al. Heterogeneous activation, local structure, and softness in supercooled colloidal liquids. Phys Rev Lett, 2019, 122: 028001

21 Mendelev MI, Kramer MJ, Ott RT, et al. Development of suitable interatomic potentials for simulation of liquid and amorphous $\mathrm{Cu}-$ Zr alloys. Philos Mag, 2009, 89: 967-987

22 Plimpton S. Fast parallel algorithms for short-range molecular dynamics. J Comput Phys, 1995, 117: 1-19

23 Nosé S. A unified formulation of the constant temperature molecular dynamics methods. J Chem Phys, 1984, 81: 511-519

24 Candelier R, Widmer-Cooper A, Kummerfeld JK, et al. Spatiotemporal hierarchy of relaxation events, dynamical heterogeneities, and structural reorganization in a supercooled liquid. Phys Rev Lett, 2010, 105: 135702

25 Smessaert A, Rottler J. Distribution of local relaxation events in an aging three-dimensional glass: Spatiotemporal correlation and 
dynamical heterogeneity. Phys Rev E, 2013, 88: 022314

26 Behler J, Parrinello M. Generalized neural-network representation of high-dimensional potential-energy surfaces. Phys Rev Lett, 2007, 98: 146401

27 Behler J. Atom-centered symmetry functions for constructing high-dimensional neural network potentials. J Chem Phys, 2011, 134: 074106

28 Cortes C, Vapnik V. Support-vector networks. Mach Learn, 1995, 20: $273-297$

29 Chang CC, Lin CJ. LIBSVM. ACM Trans Intell Syst Technol, 2011, 2: $1-27$

30 Zhang Y, Ashcraft R, Mendelev MI, et al. Experimental and molecular dynamics simulation study of structure of liquid and amorphous $\mathrm{Ni}_{62} \mathrm{Nb}_{38}$ alloy. J Chem Phys, 2016, 145: 204505

31 Sheng HW, Ma E, Kramer MJ. Relating dynamic properties to atomic structure in metallic glasses. J Minerals Metals Mater Soc, 2012, 64: 856-881

32 Slotterback S, Toiya M, Goff L, et al. Correlation between particle motion and Voronoi-cell-shape fluctuations during the compaction of granular matter. Phys Rev Lett, 2008, 101: 258001

33 Rieser JM, Goodrich CP, Liu AJ, et al. Divergence of Voronoi cell anisotropy vector: A threshold-free characterization of local structure in amorphous materials. Phys Rev Lett, 2016, 116: 088001

34 Zhang S, Liu C, Fan Y, et al. Soft-mode parameter as an indicator for the activation energy spectra in metallic glass. J Phys Chem Lett, 2020, 11: 2781-2787

35 Milkus R, Zaccone A. Local inversion-symmetry breaking controls the boson peak in glasses and crystals. Phys Rev B, 2016, 93: 094204

36 Yang J, Wang YJ, Ma E, et al. Structural parameter of orientational order to predict the boson vibrational anomaly in glasses. Phys Rev Lett, 2019, 122: 015501

Acknowledgements This work was supported by the National Natural Science Foundation of China Joint Program (U1930402), the Strategic Priority Research Program of the Chinese Academy of Sciences (XDB30000000), the National Key Research and Development Plan (2018YFA0703603), and the Natural Science Foundation of Guangdong Province (2019B030302010). Insightful discussions with Yuanchao Hu, Renzhong Li and Bin Xu are highly acknowledged. We also acknowledge the computational support from Beijing Computational Science Research Center (CSRC).

Author contributions Bai $\mathrm{H}$ and Guan P conceived and led the research; Wu Y conducted the calculations and analyzed the data. Wu Y, Guan P and Bai $\mathrm{H}$ wrote the paper with the input from Wang WH. All authors contributed to the general discussion.

Conflict of interest The authors declare that they have no conflict of interest.

Supplementary information online version of the paper.

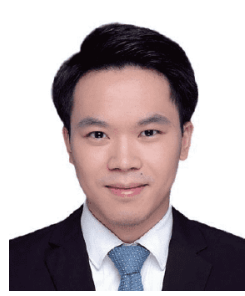

Yicheng Wu received his $\mathrm{PhD}$ degree from the Institute of Physics, Chinese Academy of Sciences, Beijing, China, in 2019. He is currently a postdoctoral fellow at Beijing Computational Science Research Center, Beijing, China. His research focuses on machine-learning and structure-property relationship in metallic glasses.

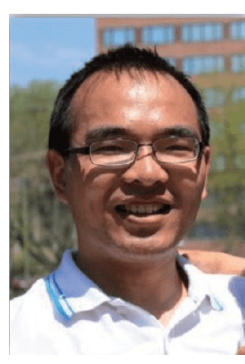

Pengfei Guan received his $\mathrm{PhD}$ degree from the Central Iron \& Steel Research Institute, Beijing, China, in 2008. He is currently a professor at Beijing Computational Science Research Center, Beijing, China. His research focuses on computational materials science towards the theoretical design of high-performance alloys, such as metallic glasses and high-strength alloys.

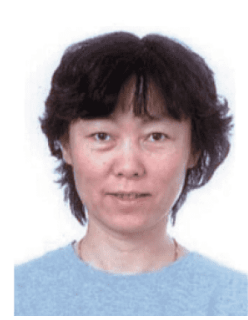

Haiyang Bai received her $\mathrm{PhD}$ degree from the Institute of Physics, Chinese Academy of Sciences, Beijing, China, in 1991. She is currently a professor at the Institute of Physics, Chinese Academy of Sciences, Beijing, China. Her research focuses on the thermodynamics, structure, and mechanical properties of metallic glasses.

\section{机器学习方法识别金属玻璃中不同动力学原子的 结构特征}

吴义成 ${ }^{1,2,3}$, 汪卫华 ${ }^{1,3,5}$, 管鹏飞 $2^{2^{*}}$, 白海洋 ${ }^{1,3,4^{*}}$

摘要 直接从无序体系的结构中预测易于发生重排的原子一直是 一个挑战. 本文以金属玻璃 $\mathrm{Cu}_{50} \mathrm{Zr}_{50}$ 为研究对象, 通过机器学习的 方法成功识别出倾向于发生重排的“类液原子”. 另外, 我们还观测 到在第一壳层以内存在一个临界尺度. 在临界尺度以内, “类液原 子”的近邻排列更紧密; 然而超过这个临界尺度, 其近邻排列相对 疏松. 研究表明“类液原子”的各向异性度更大，这可能是“类液原 子”更容易发生重排的结构起源. 我们的研究表明机器学习能为理 解金属玻璃本征的不均匀性提供一种非传统的方法, 同时揭示了 无序材料中不均匀动力学的结构起源. 